\title{
Multiple Kernel Based KNN Classifiers for Vehicle Classification
}

\author{
Pradeep Kumar Mishra \\ Ph.D Research Scholar Vision Image Processing Lab \\ Department of Electrical Engineering, Indian Institute of Technology Bombay \\ Mumbai 400076 \\ pkmishraiitb@ee.iitb.ac.in \\ Biplab Banerjee \\ Ph.D Research Scholar Image Processing Lab \\ Department of Centre of Studies Resources Engineering ,Indian Institute of Technology Bombay \\ Mumbai 400076 \\ biplab.banerjee@ee.iitb.ac.in
}

\begin{abstract}
The problem of vehicle classification has been addressed in this correspondence. Vehicle classification is a difficult task due to near similarity among various types vehicle features. Spectral properties of the image and near similarity between the front side of different vehicles makes the generalization process even more difficult. Here a multiple kernel based k-nearest neighbor classifier has been designed to improve the classification accuracy. After extracting the frames from the traffic video, vehicles are detected using background subtraction method. Then a wavelet and interest point based feature extraction step is carried out for each detected vehicle. Final classification is carried out using the newly proposed multiple kernel based k-nearest neighbor( KNN) algorithm. Experiments on several real time data-sets establish the higher accuracy of the proposed method in comparison to three well-known state of the art classification techniques.
\end{abstract}

\section{General Terms:}

KNN, Feature Extraction

\section{Keywords:}

Classification, Machine Learning, Kernel, MKL,

\section{INTRODUCTION}

The detection and classification of different vehicles classes has become a potential field of research because of its large applications in video based intelligent transportation system. Over the period of time, vehicle counting on a busy intersection helps concerned authority to efficiently control the duration of traffic signal on a road to reduce the level of traffic congestion during rush hours. This is one of the applications. To move ahead, just by counting of vehicles and their classification we can reduce the deceptive activities in many areas such as toll collection, parking area. These motion based detection and classification methods comprises interframe difference method [25] optical flow estimation [13] Gaussian scale mixture model methods [46] [28] and background subtraction methods [26] [18] [9]. Out of all the above mentioned methods background subtraction is the most common techniques used in vehicle detection. In this technique, from a given frame of video, background is subtracted. This gives a foreground blobs which matches up to the moving vehicles. Other than that, some vehicle detection methods deploy deformable 3-D geometric model [39] and graph cuts [14] object based segmentation [8] [35] to detect vehicles in motion and determine their properties. Along with all the above methods, one other method is virtual detection line method(VDL) [40]. In these methods, by using the luminance value of pixels of the moving objects, a time spatial image(TSI) is produced. These objects move on a virtual line.Each of the moving objects passing through the VDL creates a spot in time spatial image. So, the number of vehicles are counted by detection of these blobs. But there are errors in this process as well. One of the major challenges from a single Time Spatial Image is that the detection of moving image is difficult since the image of moving vehicle overlap each other. The major limitation is the occlusion. The reason behind counting errors is not only occlusion but also the morphological operations used for the generation of segmented blobs, corresponding to moving objects that are close to each other. So for minimization of error the methods mentioned in [37] [38] uses a fairly accurate width of a vehicle.This is used to take decision on the merging of blobs with the time spatial images. By analysis of motion fields of merged reactions, the merging of blobs can also be identified.

These experiments are based on the videos taken for the vehicles which are moving. Then accordingly, frames are taken and the vehicles are detected. Conventionally, feature-based algorithms are used to classify the moving objects which will also include human beings or vehicles which we can be retrieve from video [4] [19] [16] [10]. In the above mentioned algorithms, the statistical and geometric features of the pixels of segmented regions corresponding to the moving objects is considered. So in this case, the linear classifier such as k nearest neighborhood (KNN) [32] [43] non-linear classifier such as support vector machine [7] [44] [30] and neural network [34] [6] [5] are used to classify these features into known classes. There are various factors which decide the performance of vehicle classification. One of which is the initial background which is ideally estimated by trial and error based chosen thresholding of the difference frames. The segmented region for all the frames of the video are considered and the classification of features is done by using the 


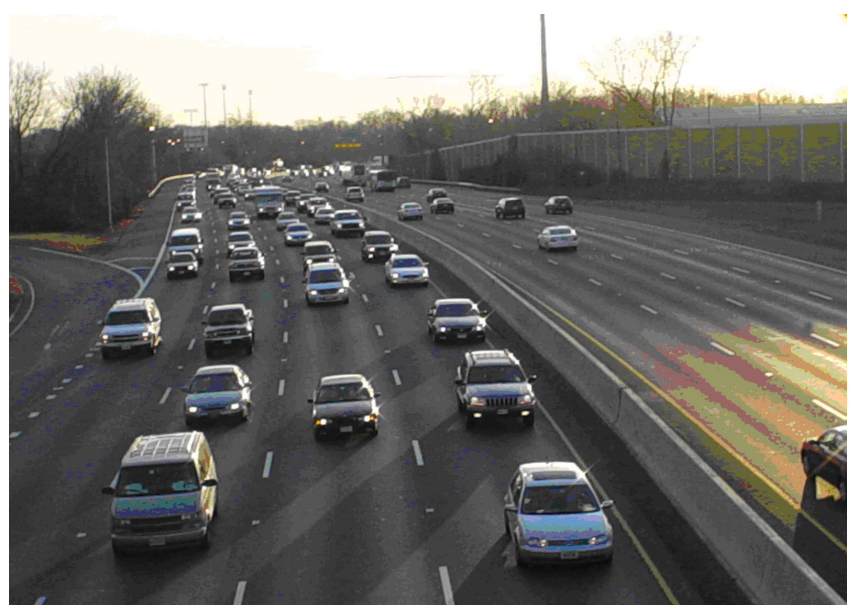

Fig. 1. Illustration of a Homogenous traffic

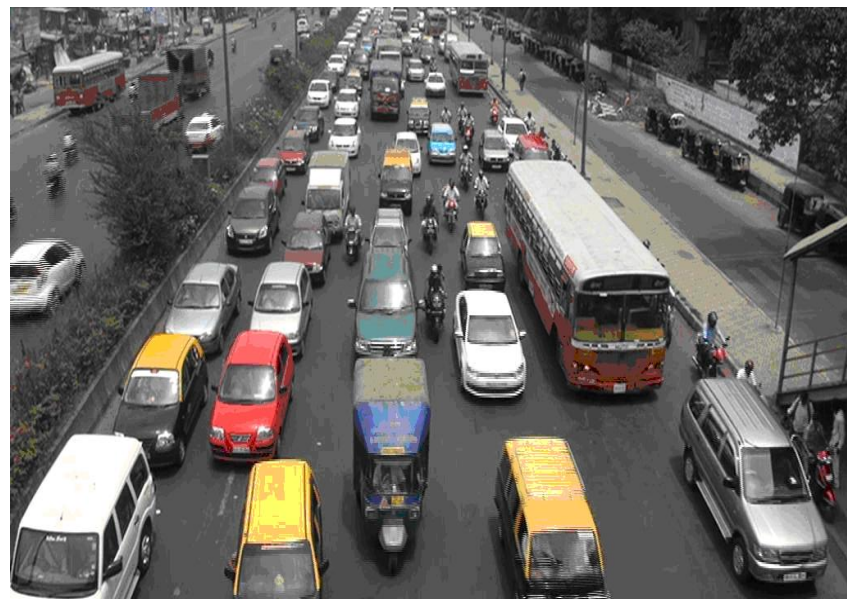

Fig. 2. Illustration of a Heterogeneous traffic

non-linear classifier. This exploits the KNN algorithms on a set of shape based features [45]. In this VDL based classifiers, the computational cost is reduced to a very great extent because it uses TSI which in turn forms a new key to frame the video. The classification and detection system becomes very robust, as the VDL method does not depend on background calculation. But the errors might increase, specifically at the peak time of traffic.

In this paper a new classification algorithm is developed which classify 2 wheeler, 3 wheeler, LMV and HMV. Taking into consideration the video input. The first step is the extraction of frame from the given video. After extraction of the frame, segmentation of the image is undertaken. After segmentation, edge detection is applied (Canny Edge). After the edge detection, morphological operation is done to form image blobs.This will give a clear image of the object to be classified. We extract the four different features from the image obtained from the above operation.These feature are explained in section 3.This forms a feature vector. Then multiple kernel KNN classifiers are applied. In this for each test point we find a similarity between test and all the training data sets and based on majority vote, classification is done accordingly.

The paper is organized as follows. In section 2 described the methodology and kernel, section 3 described feature sets and section 4 we propose classification algorithm. Section 5 gives the experimental setup and results,followed by conclusion and remarks in Section 6.

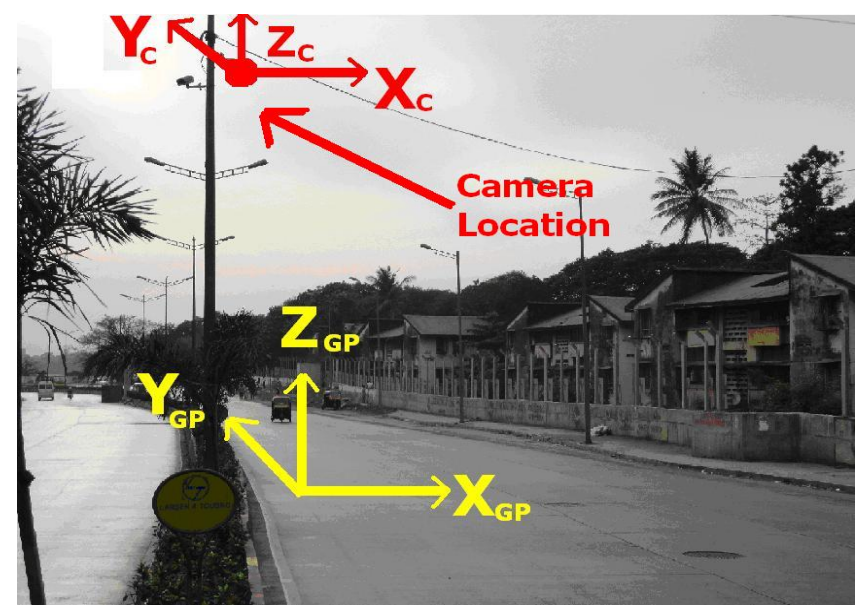

Fig. 3. Geometry at the test site near IITB campus, Mumbai.

\section{METHODOLOGY}

\subsection{The Kernel Trick}

The research on kernel methods [12] [31] has been gradually becoming an important topic because of the success of support vector machines(SVMs). It has also found many applications for pattern recognition and machine learning in various areas. In general, any kernel methods solution comprises two parts: a module that performs the mapping into the embedding or feature space and a learning algorithm designed to discover linear pattern in that space. There are two main reason why this approach should work. First of all, detecting linear relations has been focus of much research in statistics and machine learning for decades, and the resulting algorithms are both well understood and efficient. Secondly, we will see that there is computational shortcut which makes it possible to represent linear patterns efficiently in high- dimensional spaces to ensure adequate representational power. The shortcut is what we call a kernel function. The- Knearest neighbor classifier( KNN) and Gaussian classifier(GC) [22] are very often used for classification for the success of SVM and kernel, we apply multiple Kernel kNN and polynomial kernel GC in study.

The basic approach of kernel method is to insert data from the original space $R^{d}$ into a feature space $H$, a Hilbert space which has a higher dimensionality, where more effective hyper planes for classification are expected to exist in this space than the original space. As we had mentioned earlier above, the algorithms can be implemented in terms of pair wise inner product feature. Any kernel function

$$
k: R^{d} \times R^{d} \rightarrow R
$$

satisfies the characterization of kernels.

Theorem1: Characterization of kernels. a function

$$
k: X \times X \rightarrow R
$$

where $\mathrm{X}$ is a vector space.

which is either continuous or has a finite domain, can be decomposed

$$
k(x, z)=<\phi(x), \phi(z)>
$$

where $x, z \in X$ into a feature map $\phi$ into a hilbert space $F$ applied to both its arguments followed by evaluation of inner product in $F$ if and only if it satisfies the finitely positive-semidefinite property. We assume that $\mathrm{k}$ satisfies the finitely positive semidefinite property and proceed to construct a feature mapping $\phi$ into a Hilbert space for which $k$ is the kernel.

Three popular kernel functions are explained as follows. 1. linear kernel: 


$$
k(x, z)=<x, z>
$$

2.Polynomial kernel:

$$
k(x, z)=(<x, z>+1)^{p}
$$

$p \geq 0$

3.Gaussian Radial Bias Function Kernel(RBF):

$$
k(x, z)=\exp \left(\frac{-\|x-z\|^{2}}{2 \sigma^{2}}\right)
$$

$\sigma \in R-\{0\}$

where $\mathrm{x}$ and $\mathrm{z}$ are the samples in $R^{d}$

An important and significant note here is that the size of the kernel matrix is $N \times N$. This consists pair wise the information of the distance measured by suitable kernel function $\mathrm{k}$ in each entry $\mathrm{k}$ and characterization of kernels. The feature mapping 0 is an identity map if linear kernel is used, else the feature mapping is non-linear.

\subsection{Multiple Kernel Learning}

The result of SVM learning is an $\alpha$-weight linear combination of kernels with a bias b.

$$
f(x)=\operatorname{sign}\left(\sum_{i=1}^{N} \alpha_{i} y_{i} k\left(x_{i}, x\right)+b\right)
$$

where the $x_{i}, i=1$ ples $\left(y_{i} \in\{ \pm 1\}\right)$

convex combination of $\mathrm{k}$ kernels ,i.e

$$
k\left(x_{i}, x_{j}\right)=\sum_{k=1}^{k} \beta_{k} k_{k}\left(x_{i}, x_{j}\right)
$$

with $\beta_{k} \geq 0$ and $\sum_{k=1}^{k} \beta_{k}=1$ Many studies and researches on SVMs and kernels have proved that multiple kernels need to be considered. This also reveals the fact that typical learning problems involve multiple and heterogonous data sources. Also, this provides flexibility. We will also see ahead, it is a graceful way of representing results, which would also provide an in depth understanding of the application. The problem, in principle which is solved by cross validation is so called as Multiple kernel learning. Many researches have centered on efficient methods for multiple kernel learning [41]. The demerits in the kernel learning in contrast to other learning methods is that the interpretation of the result decision function is very hard, so it becomes very difficult to take out the relevant knowledge about the problem. So now try to solve the problem by convex combination of $k$ kernels. The optimized combination coefficients, for an accurate designed subkernel $K_{k}$ can be very well used to figure out the features of the examples which are of importance for discrimination: when an accurate classification is obtained by sparse weighing $\beta_{k}$, the interpretation of resulting decision function becomes easier.This significant property is not available in kernel based algorithms.One important point is that this is dissimilar to the kernel mixture framework by [33] [20] because here the kernel and each example are allotted an independent weight. So the resulting decision function interpretation becomes very hard.

\subsection{Multiple kernel based KNN classifier}

In feature space for multiple kernel $\mathrm{KNN}$,the distance measurement between training samples and test samples is difined as,

$$
\begin{gathered}
\left\|\phi\left(x^{*}\right)-\phi\left(x_{i}\right)\right\|^{2} \\
=\left(\phi\left(x^{*}\right)-\phi\left(x_{i}\right)\right)^{T}\left(\phi\left(x^{*}\right)-\phi\left(x_{i}\right)\right) \\
=\phi\left(x^{*}\right)^{T} \phi\left(x^{*}\right)-2 \phi\left(x^{*}\right)^{T} \phi\left(x_{i}\right)+\phi\left(x_{i}\right)^{T} \phi\left(x_{i}\right)
\end{gathered}
$$

where $\phi: R^{d} \rightarrow H$ is the feature mapping that embeds the data from original space $R^{d}$ into a feature space $\mathrm{H}$,and corresponding kernel function is

$$
k(x, z)=<\phi(x), \phi(z)>, x, z \in R^{d}
$$

\section{VEHICLE CLASSIFICATION}

\subsection{Feature selection}

We have used following 4 features.

(1) Haar Feature - Haar-like features are popular image features used in object recognition by [36] [2] [17] which are similar to Haar wavelets used in the first real-time face detector by Viola et al. [42]. One other contribution was to use summed area which they called integral images. Integral images can be defined as two-dimensional lookup tables in the form of a matrix with the same size as that of the original image. Each Haar-like feature may need more than four lookups, depending on how it was defined. Viola and Jones's 2-rectangle features need six lookups, 3-rectangle features need eight lookups, and 4-rectangle features need nine lookups.

(2) Pyramidal Histogram of Oriented Gradients(PHOG) - Estimating the shape of an object is often described by the edge orientations of the object.So if we calculate gradients of edges and put them in groups then we get PHOG which is used for multi class object classification as demonstrated by Bai [3] and Gehler et al. [15].Then we call each interval a 'bin' and put each pixel in a bin such that gradient of edge at that pixel lies in the interval denoted by that bin.

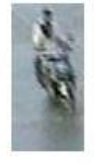

Type 1

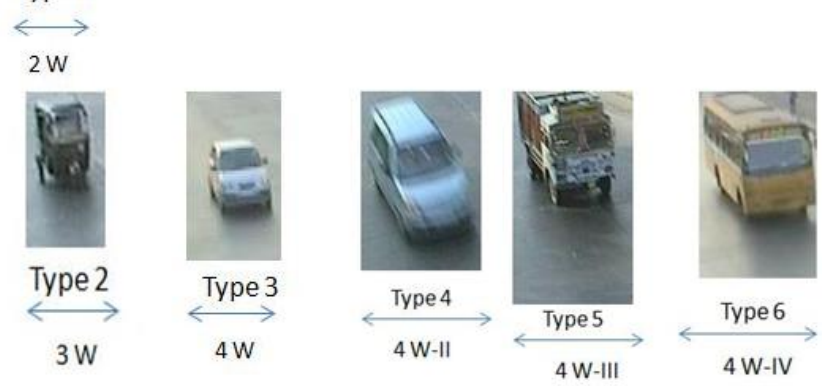

Fig. 4. Six type of vehicle are taken in the experimentation.Type1(motorbike),Type2(Autorickshaw),Type3(car), Type4(jeep),Type5(Truck),Type6(bus)

(3) Shape feature - The shape is a low level feature which describes some representations of a given object in an image. In case of a vehicle, aspect ratio seems to be useful, as it captures the appearance of a given vehicle which can be used 
for classification. Jolly et al. [11] and Kim et al. [21] use similar shape features for object classification.In this case, preference is given to six features in order to illustrate the shape of the vehicle.

1.Width- The width can be calculated based on the approximation of object blobs.

2.Area- The total number of pixels in the given cropped image will give the area.

3.Compactness-this establish the closeness to the circular shape of the vehicle, is calculated as the ratio of the area and square of perimeter of the cropped image.

4.Length- width ratio- The length to width ratio can be found from the cropped image.

(4) Scale Invarient Feature Transform- Scaleinvariant feature transform is an algorithm in computer vision to describe local features in images as shown by Lowe[23]. Lowe's method for image feature generation, transforms an image into a large collection of feature vectors, each of which is invariant to image translation, scaling, and rotation, partially invariant to illumination changes and robust to local geometric distortion. Scale invariant feature transforms image data into scale invariant coordinates relative to local features. Ma et al. [24] also uses modified SIFT descriptors for vehicle classification.

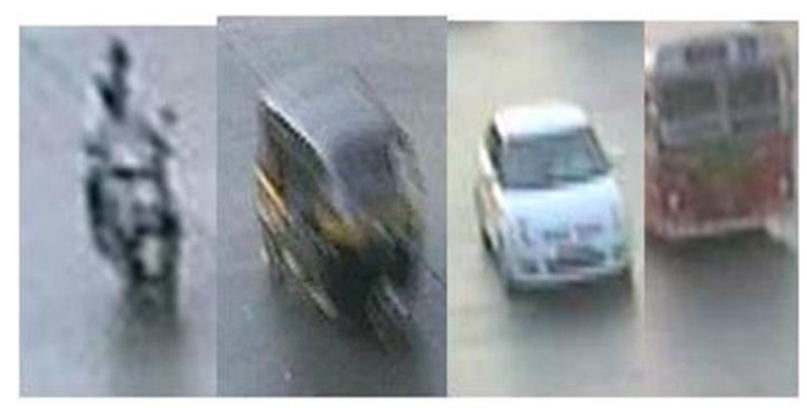

Fig. 5. Vehicle Classes. (a)Two wheeler, (b)Three wheeler ,(c)LMV,(d)HMV

In order to find these classifications for 2 wheeler, 3 wheeler, LMV and HMV, the shape based features are widely used. To be precise, car, buses, 2 wheeler and based on India scenario 3 wheeler i.e. rickshaw is used wherein the shapes are quite few shape invariant and texture based features are taken into consideration. In this anticipated method, the first, second and third order shape invariant cropped images instant are used [27]. Basically these instants are estimated from the pixel values of the cropped images. The translation, rotation and scale of the blobs have no effect on these above. In order to describe the texture of an image, the statistics of pixel values is deployed. So the data features e.g. the mean, variance, skewness, entropy of the pixel values of the mentioned cropped images, are used as textural based features. These three shaped invariant and four texture based features are exercised to discover the type of vehicle which belongs to a certain broad class of vehicles.

\section{PROPOSED CLASSIFICATION ALGORITHMS}

The proposed algorithm is developed for real time video applications. The use of this algorithm is preferred because of its low computational complexity and also better generalization accuracy.To elaborate the classification method. We have extended classical K-nearest neighbor by incorporating multiple kernel. In the proposed algorithm we find the similarity between the

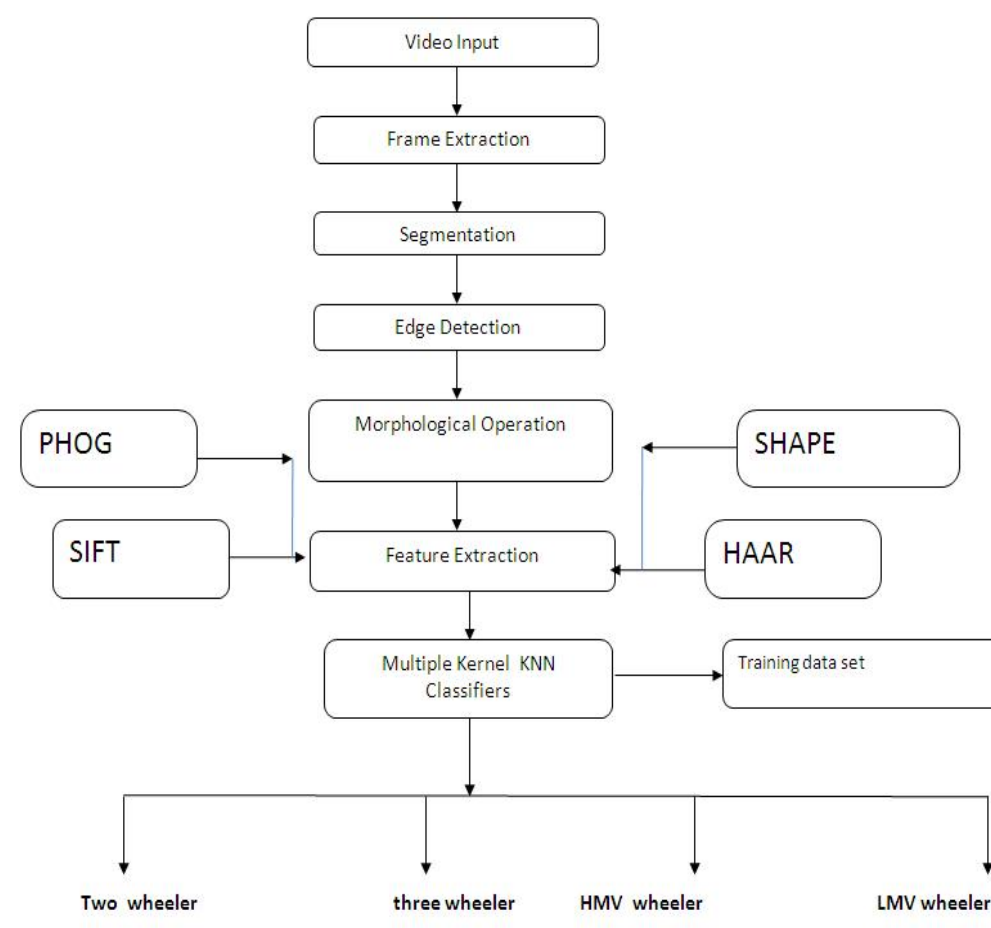

Fig. 6. Block diagram of proposed classification algorithms

Table 1. Confusion matrix for vehicle data using the Proposed method

\begin{tabular}{|l||r|r|r|c|}
\hline & 2-Wheeler & 3 -Wheeler & LMV & HMV \\
\hline \hline 2-Wheeler & 98 & 12 & 3 & 2 \\
\hline 3-Wheeler & 10 & 127 & 5 & 3 \\
\hline LMV & 4 & 9 & 142 & 15 \\
\hline HMV & 2 & 10 & 14 & 154 \\
\hline
\end{tabular}

Accuracy $=92.95 .4 \%$

training data and the test data by using multiple kernel (i.e. linear,Gaussian, Polynomial). After extracting the feature vector we work out the similarity between each test data to all the training data. Also, we decide the class by majority voting and accuracy by corresponding value of $\mathrm{k}$. The proposed classification algorithm will be very efficient in classifying different classes or types of vehicle for each of the test data points. In order to finalize the class or type of a vehicle majority voting scheme will be employed. One important note if we increase the distance between camera and vehicle,the chance of merging of blobs images will reduce. To elaborate the classification method the multiple kernel based( $\mathrm{KNN}$ ), first of all vehicle are divided into four different classes i.e 2-Wheeler,3-Wheeler,light motor vehicle(LMV) and heavy motor vehicle(HMV). We prepared all possible classes in the training data set and aslo test data set for these type of vehicles.

The proposed classification algorithm can provide different classes or type of vehicles of each of cropped image. To finalize the vehicle classes a majority voting scheme has been used. So the cropped images can be given priority based on the distance of videos from the camera which is fixed. To decide the class of vehicles we used majority voting scheme 
Table 2. Confusion matrix for vehicle data using the method[29]

\begin{tabular}{|l||r|r|r|c|}
\hline & 2-Wheeler & 3-Wheeler & LMV & HMV \\
\hline \hline 2-Wheeler & 105 & 9 & 1 & 0 \\
\hline 3-Wheeler & 5 & 132 & 8 & 0 \\
\hline LMV & 0 & 6 & 150 & 14 \\
\hline HMV & 0 & 0 & 18 & 162 \\
\hline
\end{tabular}

Accuracy $=90.0 \%$

\section{EXPERIMENTAL SETUP}

The test site for this study was setup outside the Indian Institute of Technology Bombay(IITB) campus, Mumbai, India.The camera used is Axis P5512 Network PTZ camera mounted at a height of 7.3 meters above the road surface on a lamp post in the middle of the road facing the vehicles at a pan angle $\phi$. The camera is equipped with a $1 / 4$ CCD sensor with the lens manually set at a focal length of $3.8 \mathrm{~mm}$, and a shutter time of $60 \mathrm{~Hz}$. The video is streamed employing the Motion JPEG compression scheme with a standard set resolution of $704 \times 576$ pixels and frame capture rate of $25 \mathrm{fps}$. The captured video is streamed live via the existing IITB private network to the lab where the computer used for processing is equipped with Intel core i5 processor and 4 gigabytes of RAM. We have dealt with images of vehicles of different size and shape. Primarily we considered two wheelers, three wheelers, light motor vehicles (including LMV), and heavy motor vehicles(HMV). For each class some real time images are shown in Figure 5.

The Proposed classification method is compared with three other existing method background extraction based vehicle classification methods [29],SVDL method [1] and MVDL [27]. The experiment is performed in three existing method and one proposed method in different video sequence of road traffic. The proposed classification algorithm multiple kernel based $\mathrm{KNN}$ is provided. The classification error is very low as compared to other three methods. The overall accuracy proposed in multiple kenel based KNN algorithm has been found to be $92.95 \%$ as shown in table I. The other existing method classification accuracy is shown in table II,III, IV with accuracy to be $90.0 \%, 87.7 \%$ and $85.40 \%$. In order to compare the performance of the proposed classification method based detection and classification of vehicles many experiments are already carried out with the present methods. These video clips are clicked at different locations in Mumbai near IITB campus. Also, the clips are taken under different sunlight and traffic conditions using a fixed camera as mentioned above. The total length of these video clips is more than $2 \mathrm{hr}$ in duration. To get accurate results, incoming traffic flows are considered. But this is with variation in elevation of camera, so that different appearances of similar vehicles are obtained through the video. Progressing further in the sequences, the images obtained from the video are converted to gray scale sequences having frame size of $176 \times 144$, with a frame rate of 25 frame/second. The length considered of the road and the camera position is such that the video resolution is from $1 / 6$ to $1 / 12 \mathrm{~m} / \mathrm{pixel}$. The traffic consist of the following: Type I, 2 wheeler, a motorbike, Type II, 3 wheeler, auto rickshaw, Type III: 4 wheeler, LMV a car, a covered van, Type V HMV A bus. In order to train the feature vectors we have taken 100 vehicles from each class. The value of $\mathrm{k}$ is amount of closeness. The relevant value of $\mathrm{k}$ is experimentally obtained by calculating the percentage of classification in determination of vehicles in $2 \mathrm{~W}, 3 \mathrm{~W}$ and $4 \mathrm{~W}$.

\section{CONCLUSION AND FUTURE WORK}

The current work has been done only for normal sunny day light conditions. The following steps are planned to be done to the study for future work. Improve accuracy and efficiency of the
Table 3. Confusion matrix for vehicle data using the method[1]

\begin{tabular}{|l||r|r|r|c|}
\hline & 2-Wheeler & 3-Wheeler & LMV & HMV \\
\hline \hline 2-Wheeler & 102 & 10 & 2 & 1 \\
\hline 3-Wheeler & 9 & 130 & 4 & 2 \\
\hline LMV & 2 & 8 & 146 & 14 \\
\hline HMV & 1 & 12 & 13 & 157 \\
\hline
\end{tabular}

Accuracy $=87.7 \%$

Table 4. Confusion matrix for vehicle data using the method[27]

\begin{tabular}{|l||r|r|r|c|}
\hline & 2-Wheeler & 3-Wheeler & LMV & HMV \\
\hline \hline 2-Wheeler & 98 & 12 & 3 & 2 \\
\hline 3-Wheeler & 10 & 127 & 5 & 3 \\
\hline LMV & 4 & 9 & 142 & 15 \\
\hline HMV & 2 & 10 & 14 & 154 \\
\hline
\end{tabular}

Accuracy $=85.4 \%$

system in moderate cum high density traffic. Test, modify and improve the existing system for all weather and day-night operations. Include additional categories of existing vehicle classes and also an unclassified vehicle category for special cases.

\section{REFERENCES}

[1] Claus Bahlmann, Ying Zhu, Visvanathan Ramesh, Martin Pellkofer, and Thorsten Koehler. A system for traffic sign detection, tracking, and recognition using color, shape, and motion information. In Intelligent Vehicles Symposium, 2005. Proceedings. IEEE, pages 255-260. IEEE, 2005.

[2] Hongliang Bai, Jianping $\mathrm{Wu}$, and Changpin Liu. Motion and haar-like features based vehicle detection. In MultiMedia Modelling Conference Proceedings, 2006 12th International, pages 4-pp. IEEE, 2006.

[3] Yang Bai, Lihua Guo, Lianwen Jin, and Qinghua Huang. A novel feature extraction method using pyramid histogram of orientation gradients for smile recognition. In Image Processing (ICIP), 2009 16th IEEE International Conference on, pages 3305-3308. IEEE, 2009.

[4] Lisa M Brown. View independent vehicle/person classification. In Proceedings of the ACM 2nd international workshop on Video surveillance \& sensor networks, pages 114123. ACM, 2004

[5] Olivier Chapelle, Patrick Haffner, and Vladimir N Vapnik. Support vector machines for histogram-based image classification. Neural Networks, IEEE Transactions on, 10(5):1055-1064, 1999.

[6] Olivier Chapelle, Vladimir Vapnik, Olivier Bousquet, and Sayan Mukherjee. Choosing multiple parameters for support vector machines. Machine learning, 46(1-3):131-159, 2002.

[7] Corinna Cortes and Vladimir Vapnik. Support-vector networks. Machine learning, 20(3):273-297, 1995.

[8] Rita Cucchiara, C Grana, Metal Piccardi, and A Prati. Statistic and knowledge-based moving object detection in traffic scenes. In Intelligent Transportation Systems, 2000. Proceedings. 2000 IEEE, pages 27-32. IEEE, 2000.

[9] Rita Cucchiara, Costantino Grana, Massimo Piccardi, and Andrea Prati. Detecting moving objects, ghosts, and shadows in video streams. Pattern Analysis and Machine Intelligence, IEEE Transactions on, 25(10):1337-1342, 2003. 
[10] Ross Cutler and Larry S. Davis. Robust real-time periodic motion detection, analysis, and applications. Pattern Analysis and Machine Intelligence, IEEE Transactions on, 22(8):781-796, 2000.

[11] M-P Dubuisson Jolly, Sridhar Lakshmanan, and Anil K. Jain. Vehicle segmentation and classification using deformable templates. Pattern Analysis and Machine Intelligence, IEEE Transactions on, 18(3):293-308, 1996.

[12] Theodoros Evgeniou, Charles A Micchelli, and Massimiliano Pontil. Learning multiple tasks with kernel methods. Journal of Machine Learning Research, 6(1):615, 2006.

[13] David Fleet and Yair Weiss. Optical flow estimation. In Handbook of Mathematical Models in Computer Vision, pages 237-257. Springer, 2006.

[14] Zhouyu Fu, Weiming Hu, and Tieniu Tan. Similarity based vehicle trajectory clustering and anomaly detection. In Image Processing, 2005. ICIP 2005. IEEE International Conference on, volume 2, pages II-602. IEEE, 2005.

[15] Peter Gehler and Sebastian Nowozin. On feature combination for multiclass object classification. In Computer Vision, 2009 IEEE 12th International Conference on, pages 221-228. IEEE, 2009.

[16] Surendra Gupte, Osama Masoud, Robert FK Martin, and Nikolaos P Papanikolopoulos. Detection and classification of vehicles. Intelligent Transportation Systems, IEEE Transactions on, 3(1):37-47, 2002.

[17] A Haselhoff and A Kummert. A vehicle detection system based on haar and triangle features. In Intelligent Vehicles Symposium, 2009 IEEE, pages 261-266. IEEE, 2009.

[18] Thanarat Horprasert, David Harwood, and Larry S Davis. A statistical approach for real-time robust background subtraction and shadow detection. In IEEE ICCV, volume 99 , pages 1-19, 1999.

[19] Weiming Hu, Tieniu Tan, Liang Wang, and Steve Maybank. A survey on visual surveillance of object motion and behaviors. Systems, Man, and Cybernetics, Part C: Applications and Reviews, IEEE Transactions on, 34(3):334-352, 2004.

[20] Seung-Jean Kim, Alessandro Magnani, and Stephen Boyd. Optimal kernel selection in kernel fisher discriminant analysis. In Proceedings of the 23rd international conference on Machine learning, pages 465-472. ACM, 2006.

[21] Z Kim and Jitendra Malik. Fast vehicle detection with probabilistic feature grouping and its application to vehicle tracking. In Computer Vision, 2003. Proceedings. Ninth IEEE International Conference on, pages 524-531. IEEE, 2003.

[22] Yuchun Lee. Handwritten digit recognition using k nearestneighbor, radial-basis function, and backpropagation neural networks. Neural computation, 3(3):440-449, 1991.

[23] David G Lowe. Distinctive image features from scaleinvariant keypoints. International journal of computer vision, 60(2):91-110, 2004.

[24] Xiaoxu Ma and W Eric L Grimson. Edge-based rich representation for vehicle classification. In Computer Vision, 2005. ICCV 2005. Tenth IEEE International Conference on, volume 2, pages 1185-1192. IEEE, 2005.

[25] A Neri, S Colonnese, G Russo, and P Talone. Automatic moving object and background separation. Signal Processing, 66(2):219-232, 1998.

[26] Massimo Piccardi. Background subtraction techniques: a review. In Systems, Man and Cybernetics, 2004 IEEE International Conference on, volume 4, pages 3099-3104. IEEE, 2004.
[27] Nafi Ur Rashid, Niluthpol Chowdhury Mithun, Bhadhan Roy Joy, and SM Mahbubur Rahman. Detection and classification of vehicles from a video using time-spatial image. In Electrical and Computer Engineering (ICECE), 2010 International Conference on, pages 502-505. IEEE, 2010.

[28] Carl Edward Rasmussen. The infinite gaussian mixture model. Advances in neural information processing systems, 12(5.2):2, 2000.

[29] Ehud Rivlin, Michael Rudzsky, Roman Goldenberg, Uri Bogomolov, and S Lepchev. A real-time system for classification of moving objects. In Pattern Recognition, 2002. Proceedings. 16th International Conference on, volume 3, pages 688-691. IEEE, 2002.

[30] Schölkopf, P Simard, V Vapnik, and AJ Smola. Improving the accuracy and speed of support vector machines. In Advances in Neural Information Processing Systems 9: Proceedings of the 1996 Conference [on Neural Information... Held in Denver... 1996], volume 9, page 375. The MIT Press, 1997.

[31] Bernhard Schölkopf and Christopher JC Burges. Advances in kernel methods: support vector learning. The MIT press, 1999.

[32] R Short, Keinosuke Fukunaga, et al. The optimal distance measure for nearest neighbor classification. Information Theory, IEEE Transactions on, 27(5):622-627, 1981.

[33] Sören Sonnenburg, Gunnar Rätsch, Christin Schäfer, and Bernhard Schölkopf. Large scale multiple kernel learning. The Journal of Machine Learning Research, 7:1531-1565, 2006.

[34] Donald F Specht. A general regression neural network. Neural Networks, IEEE Transactions on, 2(6):568-576, 1991.

[35] Narayan Srinivasa. Vision-based vehicle detection and tracking method for forward collision warning in automobiles. In Intelligent Vehicle Symposium, 2002. IEEE, volume 2, pages 626-631. IEEE, 2002.

[36] Zehang Sun, George Bebis, and Ronald Miller. Quantized wavelet features and support vector machines for on-road vehicle detection. In Control, Automation, Robotics and Vision, 2002. ICARCV 2002. 7th International Conference on, volume 3, pages 1641-1646. IEEE, 2002.

[37] Zehang Sun, George Bebis, and Ronald Miller. Object detection using feature subset selection. Pattern recognition, 37(11):2165-2176, 2004.

[38] Zehang Sun, George Bebis, and Ronald Miller. On-road vehicle detection using evolutionary gabor filter optimization. Intelligent Transportation Systems, IEEE Transactions on, 6(2):125-137, 2005.

[39] Zehang Sun, George Bebis, and Ronald Miller. On-road vehicle detection: A review. Pattern Analysis and Machine Intelligence, IEEE Transactions on, 28(5):694-711, 2006.

[40] Belle L Tseng, Ching-Yung Lin, and John R Smith. Realtime video surveillance for traffic monitoring using virtual line analysis. In Multimedia and Expo, 2002. ICME'02. Proceedings. 2002 IEEE International Conference on, volume 2, pages 541-544. IEEE, 2002.

[41] Manik Varma and Bodla Rakesh Babu. More generality in efficient multiple kernel learning. In Proceedings of the 26th Annual International Conference on Machine Learning, pages 1065-1072. ACM, 2009.

[42] Paul Viola and Michael Jones. Rapid object detection using a boosted cascade of simple features. In Computer Vision and Pattern Recognition, 2001. CVPR 2001. Proceedings of the 2001 IEEE Computer Society Conference on, volume 1, pages I-511. IEEE, 2001. 
[43] Kilian Q Weinberger and Lawrence K Saul. Distance metric learning for large margin nearest neighbor classification. The Journal of Machine Learning Research, 10:207-244, 2009.

[44] Jason Weston, Sayan Mukherjee, Olivier Chapelle, Massimiliano Pontil, Tomaso Poggio, and Vladimir Vapnik. Feature selection for svms. Advances in neural information processing systems, pages 668-674, 2001.
[45] Dietrich Wettschereck. A study of distance-based machine learning algorithms. 1994.

[46] Zoran Zivkovic. Improved adaptive gaussian mixture model for background subtraction. In Pattern Recognition, 2004. ICPR 2004. Proceedings of the 17th International Conference on, volume 2, pages 28-31. IEEE, 2004. 